\title{
Le philosophe qui décrit un passé dur et nous voit un avenir doux
}

\author{
Jean Martin \\ Dr med., membre de la rédaction
}

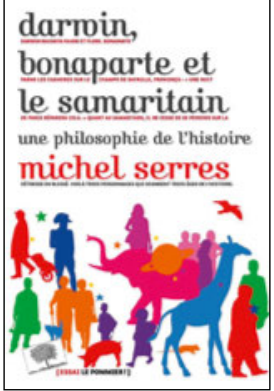

Michel Serres Darwin, Bonaparte et le Samaritain

Une philosophie de l'histoire Paris: Editions Le Pommier; 2016. 180 pages. $29.50 \mathrm{CHF}$. ISBN 978-2-746-51098-2

Sur un autre livre récent du même auteur: Martin J. La jeune génération doit tout réinventer (à propos de Petite Poucette). Bull Méd Suisses 2013, 94(38):1461-2.
Michel Serres, d'abord officier de marine, est philosophe de sciences et, à 86 ans, a derrière lui une remarquable carrière d'écrivain et d'enseignant (professeur à Stanford notamment). Intelligence encyclopédique avec une fibre écologique marquée. Son dernier ouvrage veut présenter l'histoire de la Terre et de la vie ${ }^{1}$. A la radio ou télévision, le charme de son accent du SudOuest stimule la compréhension d'une réflexion foisonnante; à l'écrit on lui recommanderait de faire plus ramassé mais il aime jouer avec une langue fleurie. «Si le sens de l'histoire aux multiples ramifications ressemble au monde tel qu'il se présente avec ses reliefs et ses choses-mémoires, ses variations saisonnières ou millénaires, alors nous pouvons célébrer de nouvelles épousailles entre l'histoire et la géographie.»

Ses chapitres décrivent trois âges: le premier est l'âge long, celui de la naissance de la Terre puis de la vie et des espèces (d'où, dans son titre, Darwin). «Une philosophie de l'histoire ne peut plus ne pas tenir compte du premier temps, colossalement long, qui contient toutes les conditions génériques du nôtre.» L'invention de l'écriture a été un fait majeur mais il est impératif d'inclure dans la réflexion ce qui s'est passé avant. «Toutes les choses autour de nous et en nous: plantes et bêtes, bactéries, roches et métaux, l'Univers, ont une histoire, sans laquelle la nôtre n'existerait point.» Le second âge est dur (d'où Bonaparte), avec la Révolution industrielle et jusqu'aux bombes d'Hiroshima et Nagasaki. A son sens, le troisième est et sera doux (d'où Le Bon Samaritain).

Il n'aime pas le mot 'environnement' qui, «avec une complaisance pathétique», laisse entendre que l'espèce humaine est le centre du monde et qu'il y a une limite précise entre nous et tout ce qui est non humain. Inévitable convivialité - ou empoignade - entre le monde et l'Homme: «Nous tenons en main le monde qui nous tient en main.»

Dur vs doux, compétition vs collaboration. Les statistiques, dit-il, "vont à l'encontre des théories prétendant que l'homme, en général, est mauvais. Partout, on trouve parmi nous plus d'empathie et de secours que de haine et de ressentiment.» "Nous devons réévaluer notre histoire, certes, mais surtout la philosophie qui la sous-tend: nos économistes enseignent comme un dogme que l'homme recherche son intérêt personnel au détriment d'autrui, lui donnant un profil de psychopathe narcissique.» Il faut refuser «l'abominable darwinisme social qui chante l'éloge du vainqueur et prône l'élimination des faibles». A propos de médecine: "Le médecin est bicéphale: une tête savante meublée de biologie et de nosographie, une autre attentive aux personnes et à leur aventure. Honneur à la tête savante, héroïne incontestée. Cependant l'autre risque d'en payer le prix, d'être oubliée.»

Enjeux. A l'ère dite anthropocène que nous vivons, "croît jusqu'à l'angoisse l'impact de nos activités, pacifiques ou mortelles, sur l'état de la planète». "Alerte rouge. La finitude et la fragilité du monde et de notre

\section{Nous tenons en main le monde qui nous tient} en main.

histoire nous obligent à nous retourner sur notre conduite et à la réformer, mieux encore, à la renverser - parce que nous vivons infinis sur un globe fini.» Une sorte de justice planétaire est indispensable. A sa dernière page: «Le Grand Récit que je tente de décrire voyage, circule, entre les paysages constellés de l'Univers, les paysages habités des vivants, les paysages génétiques des cellules, les paysages organiques de flore et de faune, enfin les paysages culturels et historiques." Basé sur son érudition, la lecture que fait Serres des évolutions récentes lui fait ainsi voir pour l'avenir un temps doux. Parmi ses composantes: «la vie prolongée par le biologiste et le médecin; la paix, nouvelle mais qui dure; les basses énergies». Sans doute peut-on observer autour de nous des manifestations de collaboration, générosité, abnégation... à côté de tant de massacres, mensonges, exploitations. Quelque peu difficile aujourd'hui de partager son optimisme mais on espère ardemment que le futur lui donne raison. 